\title{
THE ASYMPTOTIC SOLUTIONS OF CERTAIN LINEAR ORDINARY DIFFERENTIAL EQUATIONS OF THE SECOND ORDER*
}

\author{
BY \\ RUDOLPH E. LANGER
}

1. Introduction. The ordinary differential equation

$$
\frac{d^{2} v}{d s^{2}}+\lambda p_{1}(s, \lambda) \frac{d v}{d s}+\lambda^{2} p_{2}(s, \lambda) v=0,
$$

in which the coefficients $p_{i}(s, \lambda)$ are expansible in descending powers of $\lambda$ when $|\lambda|$ is large, includes as special cases many differential equations of classical importance. It is accordingly the subject of an extensive literature. In particular, the problem of the asymptotic dependence of its solutions upon the complex parameter $\lambda$ has been the subject of many investigations, in which for suitably restricted configurations a theory of considerable generality has been deduced.

A familiar change of variable gives to the equation the form

$$
\frac{d^{2} u}{d s^{2}}-\left\{\lambda^{2} q_{0}(s)+\lambda q_{1}(s)+q_{2}(s, \lambda)\right\} u=0,
$$

in which $q_{2}(s, \lambda)$ is bounded for large values of $\lambda$. The salient hypothesis, then, which has most generally been assumed and under which a developed theory is known, is that the variable $s$ be real, and that on the basic interval considered, the characteristic equation

$$
\theta^{2}-q_{0}(s)=0
$$

have roots $\theta_{i}(s)$ which are everywhere distinct. $\dagger$ In recent papers the author has studied the asymptotic forms for a type of equation not subject to this hypothesis by virtue of the fact that the coefficient $q_{0}(s)$ becomes zero at some point of the domain of the variable considered. The form of the solutions was determined, moreover, for the entire complex plane. $\ddagger$ With such a configuration of variables, the feature of prime interest was found to lie in

* Presented to the Society, December 27, 1933; received by the editors July 21, 1933.

$\dagger$ In this connection special mention is due to Birkhoff and Tamarkin. For references cf. Langer, R. E., On the asymptotic solutions of differential equations, etc., these Transactions, vol. 34 (1932), pp. 447-480.

$\ddagger$ Cf. the previous reference. 
the incidence of the Stokes' phenomenon, under which the analytic forms used for the asymptotic representation of any specific solution must be changed in a discontinuous manner as the point $(s, \lambda)$ varies across certain specifiable boundaries in the complex $(s, \lambda)$ space. This is attributable to the fact that the asymptotic representation utilizes multiple-valued expressions for the description of the single-valued solutions of the equation.

Referring to the equation in form (2) the investigation cited was made for the case in which the coefficient $q_{0}(s)$ vanishes at some point like any real positive power of the variable, but under the assumption that the term in $\lambda$ does not occur, i.e., $q_{1}(s) \equiv 0$. In the present paper the case in which the term in $\lambda$ is present while at some point the term in $\lambda^{2}$ vanishes to the second order is to be studied.* It is planned as a sequel to apply the results of this discussion to a study of the functional form of the solutions of the Mathieu equation over the complex plane, in a manner resembling that in which the earlier results were applied to a study of the Bessel's functions.

It may be of interest to note that the case to be considered bears a certain formal limiting relationship to a class of cases which by suitable transformations may be brought under the theory developed in the earlier papers cited. Thus, consider the equation

$$
\frac{d^{2} u}{d s^{2}}-\left\{\lambda^{2} q_{0}(s)+\lambda^{r} q_{1}(s)+q_{2}(s, \lambda)\right\} u=0,
$$

with

$$
q_{0}(s) \equiv s^{\nu} \sum_{j=0}^{\infty} a_{j} s^{i}, a_{0} \neq 0,
$$

and let the variable be changed by the substitution $s=\lambda^{-\sigma} z$, with $\sigma$ an undetermined positive constant. The equation takes the form

$$
\frac{d^{2} u}{d z^{2}}-\left\{\lambda^{2-2 \sigma-v \sigma} a_{0} z^{\nu}+\lambda^{2-8 \sigma-v \sigma} a_{1} z^{\nu+1}+\cdots+\lambda^{r-2 \sigma} q_{1}+q_{2}\right\} u=0,
$$

and if $r<4 / 2+\nu$ it is always possible to choose $\sigma$ so as to make the second highest power of $\lambda$ which occurs the zeroth power. Then if $-\rho^{2}$ is written for the highest power of $\lambda$, the equation takes the form

$$
\frac{d^{2} u}{d z^{2}}+\left\{\rho^{2} z^{y} a_{0}+\chi(z, \rho)\right\} u=0 .
$$

It is seen that when $\nu=2$, as in the equation (2), the transformation is possi-

* In this connection cf. for the case of a real variable Goldstein, S., A note on certain approximate solutions of linear differential equations of the second order (2), Proceedings of the London Mathematical Society, (2), vol. 33 (1932), p. 246. 
ble whenever $r<1$. For this class of equations the equation (2), in which $r=1$, may evidently be regarded as a limiting form.

2. The normal equation. In the differential equation (2) let the zero of the coefficient $q_{0}(s)$ be designated by $s_{0}$, and with $c$ as a tentatively undetermined constant let the change of variable

$$
z=s-s_{0}-\frac{c}{\lambda}
$$

be made. With the use of the formal relations

$$
\begin{aligned}
& q_{0}(s)=q_{0}\left(z+s_{0}\right)+\frac{c}{\lambda} q_{0}^{\prime}\left(z+s_{0}\right)+\frac{c^{2}}{\lambda^{2}} q_{0}^{\prime \prime}\left(z+s_{0}+\frac{\theta_{1} c}{\lambda}\right), \\
& q_{1}(s)=q_{1}\left(z+s_{0}\right)+\frac{c}{\lambda} q_{1}^{\prime}\left(z+s_{0}+\frac{\theta_{2} c}{\lambda}\right),
\end{aligned}
$$

the differential equation may then be written

$$
u^{\prime \prime}(z)-\left\{\lambda^{2} \chi_{0}^{2}(z)+\lambda \chi_{1}(z)+\chi_{2}(z, \lambda)\right\} u=0,
$$

in which, specifically,

$$
\begin{aligned}
\chi_{0}^{2}(z) & =q_{0}\left(z+s_{0}\right), \\
\chi_{1}(z) & =q_{1}\left(z+s_{0}\right)+c q_{0}^{\prime}\left(z+s_{0}\right) .
\end{aligned}
$$

Whatever choice of the constant $c$ is made, the zero of the coefficient $\chi_{0}{ }^{2}(z)$ clearly occurs at the origin. It is readily found that $c$ may always be so chosen that the relation

$$
3 \chi_{0}^{\prime}(0) \chi_{1}^{\prime}(0)-2 \chi_{0}^{\prime \prime}(0) \chi_{1}(0)=0
$$

is satisfied, and this choice will be made inasmuch as it is convenient for subsequent purposes. The equation (3) will then (i.e., when (4) is satisfied) be designated as in normal form, and the preliminary normalization of the equation will be assumed in the discussion which is to ensue.

The more specific description of the equation for which a theory is to be deduced may be given, as follows, through the means of an enunciation of the hypotheses to be made. The variable $z$ is to be complex, and is to vary over a simply connected (finite or infinite) fundamental region $R_{z}$, which includes the origin and in which the hypotheses, to be enumerated from (i) to (v) below, are simultaneously fulfilled. It may be considered as a blanket hypothesis upon the equation that some such region exists. The explicit assumptions are the following: 
(i) Within the region $R_{z}$ the coefficient $\chi_{0}{ }^{2}(z)$ is analytic and has a zero of the second order at the origin. Moreover, except in the immediate neighborhood of the origin the functions.

$$
\chi_{0}(z) \text { and } \int_{0}^{s} x_{0}(z) d s
$$

are bounded from zero.

(ii) Within the region $R_{z}$ the coefficient $\chi_{1}(z)$ is analytic, and is such that the functions

$$
\frac{\chi_{1}(z)}{x_{0}^{2}(z)} \text {, and } \frac{\int_{\alpha}^{z}\left\{\chi_{1}(z) / \chi_{0}(z)\right\} d z}{\int_{0}^{z} \chi_{0}(z) d z} \text { with } \alpha \neq 0 \text {, }
$$

are bounded except possibly in the immediate neighborhood of the origin.

(iii) The coefficient $\chi_{2}(z, \lambda)$ is analytic in $R_{z}$, and in any finite portion of $R_{z}$ is bounded uniformly when $|\lambda|$ is sufficiently large.

The enunciation of hypotheses (iv) and (v), which are less transparent, will be deferred to $\$ 7$.

For definiteness the function $\arg \chi_{0}(z)$ will be determined by the relation

$$
\arg \left\{\lim _{z \rightarrow 0} z^{-1} \chi_{0}(z)\right\}=0 .
$$

This involves no loss of generality since the adjustment may always be achieved by the transfer of a suitable constant factor from the parameter $\lambda^{2}$ to the coefficient $\chi_{0}{ }^{2}(z)$.

3. The related equation. Let the relations

$$
\begin{aligned}
k & =-\frac{\chi_{1}(0)}{4 \chi_{0}^{\prime}(0)}, \\
\eta(z) & =\frac{\chi_{1}(z)}{\chi_{0}(z)}+\frac{2 k \chi_{0}(z)}{\int_{0}^{z} \chi_{0}(z) d z},
\end{aligned}
$$

$$
\Phi(z)=\int_{0}^{z} \phi(z) d z
$$

$$
\phi(z)=2 \chi_{0}(z)+\frac{\eta(z)}{\lambda}
$$

$$
\begin{gathered}
\xi=\lambda \Phi(z), \\
\Psi(z)=\frac{\Phi^{1 / 4}(z)}{\phi^{1 / 2}(z)}
\end{gathered}
$$

serve respectively to define the symbols which occur upon the left. The function $\eta(z)$, which is analytic in $R_{z}$ because of the hypotheses (i) and (ii), is 
found to vanish at the origin in virtue of the normalization (4). It follows from this and the hypotheses that outside of any neighborhood of the origin the functions $\phi(z)$ and $\Phi(z)$ are bounded from zero uniformly when $|\lambda|$ is sufficiently large, while at the origin they have zeros of the first and second orders respectively. The function $\Psi(z)$ is accordingly analytic (with proper definition at $z \equiv 0$ ) and uniformly bounded in any finite portion of $R_{z}$. Lastly, it may be shown that since the zero of $\phi(z)$ is a simple one, the expression

$$
\omega(\phi)=\frac{-3}{16}\left(\frac{\phi}{\Phi}\right)^{2}-\frac{\phi^{\prime \prime}}{2 \phi}+\frac{3}{4}\left(\frac{\phi^{\prime}}{\phi}\right)^{2}
$$

is analytic (with proper definition at $z=0$ ).

Let $M_{\mu, \nu}(\xi)$ denote the confluent hypergeometric function customarily so designated, ${ }^{*}$ and let the functions $y_{1}(z), y_{2}(z)$ be defined by the formulas

$$
y_{j}(z)=\Psi(z) \xi^{-1 / 4} M_{k, \pm 1 / 4}(\xi), \quad j=1,2 . \dagger
$$

The functions $M$ satisfy the equation

$$
M_{\mu, \nu}^{\prime \prime}(\xi)-\left\{\frac{1}{4}-\frac{\nu}{\xi}-\frac{1-4 \mu^{2}}{4 \xi^{2}}\right\} M_{\mu, \nu}(\xi)=0,
$$

from which it may be found by direct substitution that the functions (6) are solutions of the differential equation

$$
y^{\prime \prime}(z)-\left\{\lambda^{2} \chi_{0}^{2}(z)+\lambda \chi_{1}(z)+\Omega(z, \lambda)\right\} y(z)=0,
$$

in which

$$
\Omega(z, \lambda)=\frac{\eta^{2}}{4}+\frac{2 k \chi_{0}{ }^{2} \int_{0}^{z} \eta d z}{\Phi \int_{0}^{z} \chi_{0} d z}-\frac{k\left(4 \chi_{0} \eta+\lambda^{-1} \eta^{2}\right)}{\Phi}+\omega(\phi) .
$$

This equation, (7), will be referred to briefly as the related equation. The similarity of its structure to that of the given equation (3), which is evident in so far as the coefficients of $\lambda$ and $\lambda^{2}$ are concerned, extends also to the remaining element. For with the analyticity of the expression $\omega(\phi)$ established, it is easily seen that the coefficient $\Omega(z, \lambda)$, as well as $\chi_{2}(z, \lambda)$, satisfies the hypothesis (iii).

4. The solutions of the related equation. The solutions (6) of the related equation take on and are determined by the initial values

* The formulas and notations of this and the following sections are taken from Whittaker and Watson, $A$ Course in Modern Analysis, 3d edition, Cambridge, University Press, 1920, chapter XVI.

† It is to be consistently understood that when the index $j$ is used in conjunction with double signs, then $j=1$ is to be associated with the upper signs and $j=2$ with the lower ones. 


$$
\begin{aligned}
y_{1}(0) & =0, & y_{2}(0) & =\Psi(0), \\
y_{1}^{\prime}(0) & =\frac{\lambda^{1 / 2}}{2 \Psi(0)}, & y_{2}^{\prime}(0) & =\Psi^{\prime}(0) .
\end{aligned}
$$

For values of the variable $\xi$ which are numerically small they are conveniently described by the formulas

$$
\begin{aligned}
& y_{1}(z)=\Psi(z) \xi^{1 / 2} e^{-\xi / 2}\left\{1+\frac{3-4 k}{2 \cdot 3} \xi+\cdots\right\}, \\
& y_{2}(z)=\Psi(z) e^{-\xi / 2}\left\{1+\frac{1-4 k}{1 \cdot 2} \xi+\cdots\right\} .
\end{aligned}
$$

For large values of $\xi$ they are describable by asymptotic formulas. Due to the incidence of the Stokes' phenomenon, however, such description is dependent upon the location of the variable $\xi$.

The origin, $z=0$, is an ordinary point for both the given and the related equations, and the region $R_{\mathrm{z}}$ accordingly consists of a single sheet. The relation (5e), however, maps the $z$ plane upon a Riemann surface having a simple branch point at $\xi=0$, and hence there corresponds to $R_{z}$ a two-sheeted region $R_{\xi}$ which is the domain of variation for the variable $\xi$. In this domain let the sectors $\Xi^{(h)}$ be defined by the relations

$$
\begin{aligned}
\Xi^{(h)}:\left(h-\frac{1}{2}\right) \pi+\epsilon \leqq \arg \xi \leqq\left(h+\frac{3 \pi}{2}\right) \pi-\epsilon, & \\
& h=-2,-1,0,1,
\end{aligned}
$$

$\epsilon$ denoting an arbitrarily small but positive and fixed constant. It is clear that these sectors overlap considerably and also that they completely cover the domain $R_{\xi}$. The corresponding sub-regions of $R_{z}$ will be designated by the same symbols. It is to be noted that they depend upon the parameter $\lambda$.

When $\xi$ lies in the sector $\Xi^{(0)}$ and $|\xi|$ is sufficiently large, it is known that

$$
\begin{aligned}
& M_{k, \pm 1 / 4}(\xi)=\Gamma\left(1 \pm \frac{1}{2}\right) e^{-k \pi i}\left\{\frac{1}{\Gamma\left(\frac{1}{2} \pm \frac{1}{4}-k\right)} W_{-k, 1 / 4}\left(\xi e^{-\pi i}\right)\right. \\
&\left.+\frac{i e^{ \pm \pi i / 4}}{\Gamma\left(\frac{1}{2} \pm \frac{1}{4}+k\right)} W_{k, 1 / 4}(\xi)\right\},
\end{aligned}
$$

with 


$$
W_{\mp k, 1 / 4}\left(-i \xi e^{\mp \pi i / 2}\right)=\left(-i \xi e^{\mp \pi i / 2}\right)^{\mp k} e^{ \pm \xi / 2}\left[1+\frac{E(\xi)}{\xi}\right]^{*}
$$

It may be noted that the formula as written is formally correct even in the exceptional cases when the index $k$ is a quarter of a real odd integer. The term in which the gamma function is infinite is then merely to be omitted.

When $\xi$ does not lie in $\Xi^{(0)}$ the appropriate formulas may be deduced by the use of those above in conjunction with the relations

$$
M_{k, \pm 1 / 4}(\xi)=i e^{\mp \pi i / 4} M_{-k, \pm 1 / 4}\left(\xi e^{-\pi i}\right) \text {. }
$$

From these facts it may be computed that when $|\xi|>N$, $\dagger$ and $\xi$ lies in the sector $\Xi^{(h)}$, then

$$
\begin{array}{r}
y_{i}(z)=\Psi(z) \xi^{-1 / 4} \Gamma\left(1 \pm \frac{1}{2}\right)\left\{C_{,, 1}^{(h)} \xi^{-k} e^{\xi / 2}\left[1+\frac{E(\xi)}{\xi}\right]\right. \\
\left.+C_{j, 2}^{(h)} \xi^{k} e^{-\xi / 2}\left[1+\frac{E(\xi)}{\xi}\right]\right\},
\end{array}
$$

with coefficients given by the formulas

$$
\begin{aligned}
& \Gamma\left(\frac{1}{2} \pm \frac{1}{4}-k\right) C_{j, 1}^{(h)}= \begin{cases} \pm i e^{-2 k \pi i}, & \text { for } h=-2, \\
1, & \text { for } h=-1, \text { or } 0, \\
\mp i e^{2 k \pi i}, & \text { for } h=1 ;\end{cases} \\
& \Gamma\left(\frac{1}{2} \pm \frac{1}{4}+k\right) C_{j, 2}^{(h)}= \begin{cases}-i e^{(k \mp 1 / 4) \pi i}, & \text { for } h=-2, \text { or }-1, \\
i e^{-(k \mp 1 / 4) \pi i}, & \text { for } h=0, \text { or } 1 .\end{cases}
\end{aligned}
$$

It is to be understood that if $k$ is such that one of the gamma functions is infinite, then the coefficient $C$ which multiplies it in (11) is to be assigned the value zero. The formulas (10) differ for different values of $h$. It is readily observed, however, that in any region common to two of the sectors $\Xi^{(i)}$ their difference is asymptotically negligible. When it is a matter of choice as to which sector is to be considered as containing the point $\xi$, then the choice is always immaterial.

The formulas

$$
y_{h, j}(z)=\mp\left\{\Gamma\left(-\frac{1}{2}\right) C_{\dot{2}, 8-j}^{(h)} y_{1}(z)+\Gamma\left(\frac{1}{2}\right) C_{1,8-j}^{(h)} y_{2}(z)\right\},
$$

which are found to have the inverse form

* The symbol $E$ will be used consistently to designate some function which is bounded. There is to be no implication that the symbol denotes the same function in different instances.

The formula given for $M_{\mu, \nu}$ by Whittaker and Watson, p. 346, appears to be in error.

$\dagger$ The symbolism $|\xi|>N$ is to be interpreted merely as an abbreviation of the statement when $|\xi|$ is sufficiently large. 


$$
y_{j}(z)=\Gamma\left(1 \pm \frac{1}{2}\right)\left\{C_{j, 1}^{(h)} y_{h, 1}(z)+C_{j, 2}^{(h)} y_{h, 2}(z)\right\},
$$

define for each index $h$ a set of solutions alternative to those described above. When $\xi$ lies in the sector $\Xi^{(m)}$ they are found to be of the forms

$$
\begin{aligned}
& y_{h, j}(z)=\Psi(z) \xi^{-1 / 4}\left\{A_{j, 1}^{(h, m)} \xi^{-k} e^{\xi / 2}\left[1+\frac{E(\xi)}{\xi}\right]\right. \\
&\left.+A_{j, 2}^{(h, m)} \xi^{k} e^{-\xi / 2}\left[1+\frac{E(\xi)}{\xi}\right]\right\},
\end{aligned}
$$

with

$$
\begin{aligned}
& A_{j, 1}^{(h, m)}= \pm \pi\left\{C_{2,3-j}^{(h)} C_{1,1}^{(m)}-C_{1,3-j}^{(h)} C_{2,1}^{(m)}\right\} \\
& A_{j, 2}^{(h, m)}= \pm \pi\left\{C_{2,8-j}^{(h)} C_{1,2}^{(m)}-C_{1,3-j}^{(h)} C_{2,2}^{(m)}\right\}
\end{aligned}
$$

These formulas reduce when $m=h$ to give

$$
\begin{aligned}
& y_{h, j}(z)=\Psi(z) \xi^{\mp k-1 / 4} e^{ \pm \xi / 2}\left[1+\frac{E(\xi)}{\xi}\right], \text { for } \xi \text { in } \Xi^{(h)}, \\
& y_{h, j}^{\prime}(z)=\frac{ \pm \lambda^{1 / 2}}{\Psi(z)} \xi^{\mp k+1 / 4} e^{ \pm \xi / 2}\left[1+\frac{E(\xi)}{\xi}\right],
\end{aligned}
$$

and in the simplicity of these forms lies the advantage of the solutions in question.

From the formulas (13a), and the fact that the Wronskian of any pair of solutions is independent of $z$, it is found directly that

$$
W\left(y_{h, 1} y_{h, 2}\right) \equiv \lambda^{1 / 2} \text {. }
$$

5. Formal developments. Let the function $\theta(z)$ be defined by the relation

$$
\theta(z)=\chi_{2}(z, \lambda)-\Omega(z, \lambda) .
$$

Then the given equation may be written in the form

$$
u^{\prime \prime}(z)-\left\{\lambda^{2} \chi_{0}^{2}(z)+\lambda \chi_{1}(z)+\Omega(z, \lambda)\right\} u(z)=\theta(z) u(z),
$$

and is accordingly solved by any function $u(z)$ which satisfies the relation

$$
u(z)=y(z)+\frac{1}{\lambda^{1 / 2}} \int_{*}^{z}\left\{y_{h, 1}(z) y_{h, 2}\left(z_{1}\right)-y_{h, 2}(z) y_{h, 1}\left(z_{1}\right)\right\} \theta\left(z_{1}\right) u\left(z_{1}\right) d z_{1} .
$$

In this $y(z)$ may be any solution of the related equation and the integration may be extended over any desired path which extends from any fixed point * to the variable point $z$. To each choice of these elements there evidently cor- 
responds a solution $u$. The correspondence between a solution $u$ and the associated function $y$ will be indicated by the use of the same subscripts upon the former as may be attached to the latter.

From the relations (5e) and (5f) it follows that

$$
d z=\frac{\Psi^{2}(z)}{\lambda^{1 / 2} \xi^{1 / 2}} d \xi,
$$

and hence if for any values of the arguments involved the functions $Q$ are defined by the formulas

$$
Q_{j}(\alpha, \beta, \delta)= \pm \theta\left(z_{1}\right) \Psi^{2}\left(z_{1}\right)\left\{\alpha_{h, j}(z) \beta_{h, 3-j}\left(z_{1}\right)-\alpha_{h, 8-j}(z) \beta_{h, j}\left(z_{1}\right) \delta\right\},
$$

the relation (15) may be written

$$
u(z)=y(z)+\frac{1}{\lambda} \int_{*}^{z} Q_{j}(y, y, 1) u\left(z_{1}\right) \frac{d \xi_{1}}{\xi_{1}^{1 / 2}} .
$$

It should be observed that differentiation of this formula leads simply to the associated one

$$
u^{\prime}(z)=y^{\prime}(z)+\frac{1}{\lambda} \int_{*}^{z} Q_{j}\left(y^{\prime}, y, 1\right) u\left(z_{1}\right) \frac{d \xi_{1}}{\xi_{1}^{1 / 2}} .
$$

When the form of $u(z)$ has been derived, this formula serves readily to yield the form of $u^{\prime}(z)$.

For the case in which the formula (17) is to be applied to the particular solutions $u_{h, j}(z)$ associated with the related solutions $y_{h, j}(z)$ it is convenient to define the symbols $U$ and $Y$ by the formulas

$$
U_{h, j}(z)=\frac{\xi^{ \pm k+1 / 4}}{\Psi(z)} e^{\mp \xi / 2} u_{h, j}(z), \quad Y_{h, j}^{(n)}(z)=\frac{\xi^{ \pm k+1 / 4}}{\Psi(z)} e^{\mp \xi / 2} y_{h, j}^{(n)}(z),
$$

in which $y^{(0)}(z) \equiv y(z)$, and $y^{(n)}(z)$ for $n>0$ is to be subsequently defined. Then formula (17) may be given in either one of the forms

$$
\begin{aligned}
& u_{h, j}(z)=y_{h, j}(z)+I\left(u_{h, j}, *, \xi\right), \\
& U_{h, j}(z)=Y_{h, j}^{(0)}(z)+J\left(U_{h, j}, *, \xi\right),
\end{aligned}
$$

in which the final terms may each again be given by alternative formulas thus:

$$
I(u, *, \xi)\left\{\begin{array}{l}
=\frac{1}{\lambda} \int_{*}^{\xi} Q_{j}(y, y, 1) u\left(z_{1}\right) \frac{d \xi_{1}}{\xi_{1}^{1 / 2}}, \\
=\frac{1}{\lambda} \int_{*}^{\xi} Q_{j}\left(y, Y^{(0)}, \xi_{1}^{\mp 2 k} e^{ \pm \xi_{1}}\right) \Psi^{2}\left(z_{1}\right) U\left(z_{1}\right) \frac{d \xi_{1}}{\xi_{1}},
\end{array}\right.
$$


and

$$
J(U, *, \xi)\left\{\begin{array}{l}
=\frac{1}{\lambda} \int_{*}^{\xi} Q_{j}\left(Y^{(0)}, y, \xi^{ \pm 2 k} e^{\mp \xi}\right) u\left(z_{1}\right) \frac{d \xi_{1}}{\xi_{1}^{1 / 2}}, \\
=\frac{1}{\lambda} \int_{*}^{\xi} Q_{j}\left(Y^{(0)}, Y^{(0)}, \xi^{ \pm 2 k} \xi_{1}^{\mp 2 k} e^{\mp\left(\xi-\xi_{1}\right)}\right) \Psi^{2}\left(z_{1}\right) U\left(z_{1}\right) \frac{d \xi_{1}}{\xi_{1}} .
\end{array}\right.
$$

The process of iteration, familiar from the theory of integral equations, may be applied to the equations (17) or (20). It leads formally to the relations

$$
u(z)=y(z)+\sum_{n=1}^{\infty} y^{(n)}(z), \quad U(z)=Y^{(0)}(z)+\sum_{n=1}^{\infty} Y^{(n)}(z),
$$

with terms given by the recurrence formulas

$$
\begin{aligned}
& y^{(n)}(z)=I\left(y^{(n-1)}, *, \xi\right), \\
& Y^{(n)}(z)=J\left(Y^{(n-1)}, *, \xi\right) .
\end{aligned}
$$

It is to be shown in the following that the series in (23) which have been obtained formally are convergent under appropriate circumstances, so that actual solutions are represented by them.

6. The solutions $u_{j}(z)$ when $|\xi| \leqq N$. With any choice of the constant $N$ the region $|\xi| \leqq N$ lies entirely within the domain $R_{\xi}$, provided $|\lambda|$ is sufficiently large. Hence the straight line from the origin to any point of the region lies within $R_{\xi}$, and may be chosen as the path of integration in the formulas (17) and (18). With this choice and with the rôle of $y(z)$ taken by $y_{j}(z)$, either one of the functions (6), the corresponding solution $u_{j}(z)$ takes at $\xi=0$, i.e., at $z=0$, the same values as $y_{j}$. Hence, from (8),

$$
\begin{aligned}
u_{1}(0) & =0, & u_{2}(0) & =\Psi(0), \\
u_{1}^{\prime}(0) & =\frac{\lambda^{1 / 2}}{2 \Psi(0)}, & u_{2}^{\prime}(0) & =\Psi^{\prime}(0) .
\end{aligned}
$$

With $\xi$ bounded the variables $z$ and $\xi_{1}$ are likewise bounded. The boundedness of the functions $y_{h, j}(z)$ and hence of the expression $Q_{j}(y, y, 1)$ follows directly, and accordingly formulas (24) and (21) yield the relation

$$
\left|y_{j}^{(n)}(z)\right| \leqq \frac{M}{|\lambda|} \int_{0}^{\xi}\left|y_{j}^{(n-1)}\left(z_{1}\right) \frac{d \xi_{1}}{\xi_{1}^{1 / 2}}\right|^{\dagger}
$$

Since $y_{j}{ }^{(0)}(z)$ is bounded it follows directly by induction that for any index $n$

$\dagger$ It is to be understood that $M$ is used merely to denote some sufficiently large constant. 


$$
\left|y_{i}^{(n)}(z)\right| \leqq M\left\{\frac{M}{|\lambda|}\right\}^{n} .
$$

The series in the formula (23) accordingly converges for sufficiently large values of $|\lambda|$, and is in fact of the order $O\left(|\lambda|^{-1}\right)$.

The conclusion thus deduced, together with that which immediately follows from (18) since $y_{h_{, j}^{\prime}}(z) \lambda^{-1 / 2}$, and hence $Q_{j}\left(y^{\prime}, y, 1\right) \lambda^{-1 / 2}$, are bounded, is the following, i.e., that

$$
\begin{aligned}
& u_{j}(z)=y_{j}(z)+\frac{E(z, \lambda)}{\lambda}, \\
& u_{j}^{\prime}(z)=y_{j}^{\prime}(z)+\frac{E(z, \lambda)}{\lambda^{1 / 2}}, \text { when }|\xi| \leqq N .
\end{aligned}
$$

7. Additional hypotheses. When $\xi$ is not restricted to be numerically bounded the considerations are less simple than those of $\$ 6$. In particular some stipulations restricting the configuration of the domain of the variables must be made, and will be framed in the following way.

A sub-region of $R_{\xi}$ will be styled as a region of the type $r$ if it is simply connected (finite or infinite), and fulfills the following specifications:

(a) that it contains the origin and lies entirely within some one of the half planes bounded by the axis $\Re(\xi)=0$;

(b) that it contains no more than one segment of any line on which $\Re(\xi)$ is constant.

Concerning a region of this type, $r$, it will be observed that it invariably contains upon its boundary (possibly at infinity if the region is infinite) a specific point $\xi_{m}$, so located that there passes through each point $\xi$ of the region an ordinary curve, $\Gamma$, which joins the origin with the point $\xi_{m}$, and upon which the abscissa, $\Re(\xi)$, varies monotonically with the arc length (in the sense of non-decreasing or non-increasing). The symbol $\Gamma$ will be reserved for the designation of arcs of curves of the type described.

As a hypothesis upon the fundamental domain $R_{z}$, and upon the range of the parameter $\lambda$, it will be assumed that

(iv) The region $R_{z}$ is such that for each admissible value of $\lambda$ every point of the corresponding region $R_{\xi}$ lies in some sub-region of the type $r$.

If the domain $R_{z}$ is finite no additional assumptions respecting the given equation need be made. However, if the domain is infinite the discussion to follow necessitates the further and final hypothesis, i.e., that 
(v) For all arcs upon which $|z| \geqq A$ ( $A$ an arbitrarily large but fixed constant) and which for some admitted value of $\lambda$ correspond to a curve of the type $\Gamma$, a relation

is uniformly satisfied.

$$
\int\left|\frac{\theta(z)}{\phi(z)} d z\right|<M
$$

8. The solutions $u_{h, j}(z)$. Let any region of the type $r$ be considered and let the index $h$ be determined by a (any) sector $\Xi^{(h)}$ which contains the region in question. With $y(z)$ in the formula (17) replaced by $y_{h, j}(z)$ the relation assumes the form (20) in which the limit of integration * still remains to be specified. This limit will be chosen as either $*=\xi_{m}$ or $*=0$ as dictated by the requirement that in proceeding along a $\Gamma$ curve from the point $*$ to the point $\xi$ the abscissa shall be algebraically non-decreasing in the case $j=1$, and non-increasing in the case $j=2$. Inasmuch as the reasoning is entirely similar the discussion will be given only for the case $j=1$. Then when the chosen region is one in which $\Re(\xi)>0, *=0$, while if $\Re(\xi)<0$ in $r$, then $*=\xi_{m}$.

Case 1. $\Re(\xi)>0$ in $r$. Since the integration extends from 0 to $\xi$, it is clear that the discussion of $\S 6$ applies without modification if $|\xi| \leqq N$. Hence

$$
\left|y_{h, 1}^{(n)}(z)\right| \leqq M\left\{\frac{M}{|\lambda|}\right\}^{n}, \text { when }|\xi| \leqq N .
$$

When $|\xi|>N$ the path of integration, which may be chosen as a $\Gamma$ curve, contains a point $\xi_{0}$ for which $\left|\xi_{0}\right|=N$. Then let the relation (24) in its second form be written

$$
Y_{h, 1}^{(n)}(z)=J\left(Y_{h, 1}^{(n-1)}, 0, \xi_{0}\right)+J\left(Y_{h, 1}^{(n-1)}, \xi_{0}, \xi\right) .
$$

The formulas (13a) and (19) show directly that when $|\xi|$ is large the functions $Y_{h, j}^{(0)}(z)$ are bounded. Since in the assumed configuration the quantities $\xi^{2 k} e^{-\xi}$ and $\left(\xi \xi_{1}^{-1}\right)^{2 k} e^{-\left(\xi-\xi_{1}\right)}$ are likewise bounded, it is clear from the first and second of the formulas (22) respectively that

$$
\begin{aligned}
& \left|J\left(Y_{h, 1}^{(n-1)}, 0, \xi_{0}\right)\right|<\frac{M}{|\lambda|} \int_{0}^{\xi_{0}}\left|y_{h, 1}^{(n-1)}\left(z_{1}\right) \frac{d \xi_{1}}{\xi_{1}^{1 / 2}}\right|, \\
& \left|J\left(Y_{h, 1}^{(n-1)}, \xi_{0}, \xi\right)\right|<\frac{M}{|\lambda|} \int_{\xi_{0}}^{\xi}\left|Y_{h, 1}^{(n-1)}\left(z_{1}\right)\right| \cdot\left|\theta\left(z_{1}\right) \Psi^{4}\left(z_{1}\right) \frac{d \xi_{1}}{\xi_{1}}\right| .
\end{aligned}
$$

Since

$$
\theta\left(z_{1}\right) \Psi^{4}\left(z_{1}\right) \frac{d \xi_{1}}{\xi_{1}}=\frac{\theta\left(z_{1}\right)}{\phi\left(z_{1}\right)} d z_{1}
$$


it follows, in virtue of hypothesis (v), that when $n=1$ the integral on the right of the first of the relations (29) is bounded while that in the second relation is of the order $O(\log |\lambda|)$. For this value of $n$, therefore, the result

$$
\left|Y_{h, 1}^{(n)}(z)\right|<M\left\{\frac{M \log |\lambda|}{|\lambda|}\right\}^{n}
$$

may be concluded, and in virtue of (27) the general validity of the relation (30) follows by induction.

Case 2. $\Re(\xi)<0$ in $r$. In this case the integration extends from $\xi_{m}$ to the point $\xi$ : If $|\xi|>N$ the formula

$$
Y_{h, 1}^{(n)}(z)=J\left(Y_{h, 1}^{(n-1)}, \xi_{m}, \xi\right)
$$

may be used, and since the right member is of precisely the structure of the final term of the relation (28) the conclusion (30) may be reached as in the preceding case.

If $|\xi| \leqq N$ the first of the formulas (24) may be written

$$
y_{h, 1}^{(n)}(z)=I\left(y_{h, 1}^{(n-1)}, \xi_{0}, \xi\right)+I\left(y_{h, 1}^{(n-1)}, \xi_{m}, \xi_{0}\right) .
$$

With the present configuration the quantity $\xi_{1}^{-2 k} e^{\xi_{1}}$ is bounded, and the formulas (21) show respectively that

$$
\begin{aligned}
& \left|I\left(y_{h, 1}^{(n-1)}, \xi_{0}, \xi\right)\right|<\frac{M}{|\lambda|} \int_{\xi_{0}}^{\xi}\left|y_{h, 1}^{(n-1)}\left(z_{1}\right) \frac{d \xi_{1}}{\xi_{1}{ }^{1 / 2}}\right|, \\
& \left|I\left(y_{h, 1}^{(n-1)}, \xi_{m}, \xi_{0}\right)\right|<\frac{M}{|\lambda|} \int_{\xi_{m}}^{\xi_{0}}\left|Y_{h, 1}^{(n-1)}\left(z_{1}\right)\right| \cdot\left|\theta\left(z_{1}\right) \Psi^{4}\left(z_{1}\right) \frac{d \xi_{1}}{\xi_{1}}\right| .
\end{aligned}
$$

In the manner of the preceding discussion, it follows readily that

$$
\left|y_{h, 1}^{(n)}(z)\right|<M\left\{\frac{M \log |\lambda|}{|\lambda|}\right\}^{n} \text {, when }|\xi| \leqq N .
$$

This relation obviously displaces (27) and is, therefore, valid for all regions $r$ in which $|\xi| \leqq N$.

The results (30) and (31) assure the convergence of the series which occur in the formulas (23), when $|\lambda|$ is large. Recalling the relations (19) the results (together with those for the derived functions and for the case $j=2$ ) may be enunciated as follows.

Corresponding to any region of the type $r$ there exists a pair of solutions of the given differential equation which in that region are subject to the descriptions 


$$
\begin{aligned}
& u_{h, j}(z)=y_{h, j}(z)+\frac{E(z, \lambda) \log \lambda}{\lambda}, \\
& u_{h, j}^{\prime}(z)=y_{h, j}^{\prime}(z)+\frac{E(z, \lambda) \log \lambda}{\lambda^{1 / 2}}, \text { when }|\xi| \leqq N,
\end{aligned}
$$

and

$$
\begin{aligned}
& u_{h, j}(z)=y_{h, j}(z)+\Psi(z) \xi^{\mp k-1 / 4} e^{ \pm \xi / 2} \frac{E(z, \lambda) \log \lambda}{\lambda}, \\
& u_{h, j}^{\prime}(z)=y_{h, j}^{\prime}(z)+\frac{\xi^{\mp k+1 / 4}}{\Psi(z)} e^{ \pm \xi / 2} \frac{E(z, \lambda) \log \lambda}{\lambda^{1 / 2}}, \text { when }|\xi|>N .
\end{aligned}
$$

It may be noted in connection with these formulas that in any region $r$ within which $|\Re(\xi)|$ is unbounded the solution of the sub-dominant form is unique except for a constant factor. This follows from the fact that every solution $u(z)$ must be expressible in the form

$$
u(z) \equiv \delta_{1} u_{h, 1}(z)+\delta_{2} u_{h, 2}(z),
$$

with coefficients which are free from $z$, and unless the coefficient of the dominant solution on the right is zero the solution is itself of the dominant form.

It should also be remarked that each set of solutions $u_{h, j}(z)$ of the description (33) has been deduced for a specific region $r$, and, although the notation has not been designed to indicate the fact, this set of solutions for any one region $r_{1}$ is not, in general, identical with that for another region $r_{2}$. In a special but important case the existence of a set of solutions which retain the forms (32), (33) over two abutting regions $r$ may be deduced as follows. Let $r_{1}$ and $r_{2}$ be two regions of this type which lie within one and the same sector $\Xi^{(h)}$ and which abut along the imaginary $\xi$ axis, $r_{1}$ lying in the half-plane $\Re(\xi) \leqq 0$, and $r_{2}$ in the half-plane $\Re(\xi) \geqq 0$. Denote by $u_{h, 1}(z)$ the solution which is sub-dominant in $r_{1}$ and by $u_{h, 2}(z)$ the solution which is sub-dominant in $\boldsymbol{r}_{2}$. These solutions are linearly independent, as may be seen by comparing the respective formulas (33) along the imaginary axis. Hence $u_{h, 1}(z)$ and $u_{h, 2}(z)$ are of the dominant form respectively in the regions $r_{2}$ and $r_{1}$, and are thus described by the formulas (32) and (33) over the two regions $r_{1}$ and $r_{2}$ simultaneously. Such a pair of regions may constitute a large portion or even the whole of the region $\Xi^{(h)}$.

9. The solutions for general values of $\xi$. Between any three of the various solutions $u(z)$ which have been defined there exists a linear relation with constant coefficients. Thus, in particular, for each index $h$

$$
u_{h, \jmath}(z) \equiv \alpha_{1, j}^{(h)} u_{1}(z)+\alpha_{2, j}^{(h)} u_{2}(z), \quad j=1,2,
$$


with coefficients given by the familiar formulas

$$
\alpha_{j, i}^{(h)}=\mp \frac{W\left(u_{3-j}, u_{h, i}\right)}{W\left(u_{1}, u_{2}\right)}, \quad i, j=1,2 .
$$

Since the Wronskians are all independent of $z$, they may be evaluated at the origin, and in virtue of the relations (8), (25), and (32) it is found that

$$
\alpha_{j, i}^{(h)}=\left\{\mp \frac{W\left(y_{3-i}, y_{h, i}\right)}{W\left(y_{1}, y_{2}\right)}+\frac{E(\lambda) \log \lambda}{\lambda}\right\} .
$$

On the other hand, the relations (12) may be made to yield the equalities

$$
\mp \frac{W\left(y_{8-i}, y_{h, i}\right)}{W\left(y_{1}, y_{2}\right)}=(-1)^{i} \Gamma\left(\mp \frac{1}{2}\right) C_{z-i, 3-i}^{(h)},
$$

whence it follows that

$$
\alpha_{i, i}^{(h)}=(-1)^{i} \Gamma\left(\mp \frac{1}{2}\right)\left\{C_{3-j, 3-i}^{(h)}+\frac{E(\lambda) \log \lambda}{\lambda}\right\},
$$

the coefficients on the right being given by the formulas (11).

With the values (35) the relations inverse to (34) are readily computed to be

$$
\begin{array}{r}
u_{j}(z)=\Gamma\left(1 \pm \frac{1}{2}\right)\left\{u_{h, 1}(z)\left\{C_{j, 1}^{(h)}+\frac{E(\lambda) \log \lambda}{\lambda}\right\}\right. \\
\left.+u_{h, 2}(z)\left\{C_{j, 2}^{(h)}+\frac{E(\lambda) \log \lambda}{\lambda}\right\}\right\},
\end{array}
$$

a result which is obtainable independently of the value of $h$. Let any point $z$ be given then, and let $h$ be determined as the index of the region $\Xi^{(h)}$ in which the point $z$ lies. The formula (36) may then be resorted to, and since for the value of $z$ given the formulas (33) and (13a) are valid, the asymptotic expression for $u_{j}(z)$ is obtained. Quantitatively the results may be summarized as follows.

The solutions of the given differential equation which are determined by the initial values (25) are given when $|\xi| \leqq N$ by the formulas

$$
\begin{aligned}
u_{j}(z) & =\frac{1}{\lambda^{1 / 4} \phi^{1 / 2}(z)} M_{k, \pm 1 / 4}(\xi)+\frac{E(z, \lambda)}{\lambda} \\
u_{i}^{\prime}(z) & =\lambda^{3 / 4} \phi^{1 / 2}(z)\left\{M_{k, \pm 1 / 4}^{\prime}(\xi)-\frac{\phi^{\prime}(z)}{2 \lambda \phi^{2}(z)} M_{k, \pm 1 / 4}(\xi)\right\}+\frac{E(z, \lambda)}{\lambda^{1 / 2}} .
\end{aligned}
$$


When $|\xi|>N$ and $\xi$ lies in a sector $\Xi^{(h)}$, then

(38)

$$
u_{j}(z)=\frac{\Gamma\left(1 \pm \frac{1}{2}\right)}{\lambda^{1 / 4} \phi^{1 / 2}(z)}\left\{\left[C_{j, 1}^{(h)}\right] \xi^{-k} e^{\xi / 2}+\left[C_{j, 2}^{(h)}\right] \xi^{k} e^{-\xi / 2}\right\},
$$

$$
u_{j}^{\prime}(z)=\lambda^{8 / 4} \phi^{1 / 2}(z) \Gamma\left(1 \pm \frac{1}{2}\right)\left\{\left[C_{j, 1}^{(h)}\right] \xi^{-k} e^{\xi / 2}-\left[C_{j, 2}^{(h)}\right] \xi^{k} e^{-\xi / 2}\right\},
$$

in which the coefficients are given by the formulas (11), and the symbol $[C]$ designates in each case an expression which differs from $C$ by quantities of the order $O\left(|\xi|^{-1}\right)$ and $O(\log |\lambda| /|\lambda|)$.

Lastly, on substituting the forms (38) into the relation (34) (the values of $h$ in the two expressions being not necessarily the same) the forms of the solutions $u_{h, j}(z)$ for general values of $z$ may be derived. These results are the following:

For any index $h=-2,-1,0$, or 1 , there exists for the given differential equation a pair of solutions $u_{h, j}(z)$, which, when $|\xi|>N$ and $z$ lies in a region, (or a pair of such regions which abut along $\Re(\xi)=0$ ) in the domain $\Xi^{(h)}$, are of the form

$$
\begin{aligned}
& u_{h, j}(z)=\frac{1}{\lambda^{1 / 4} \phi^{1 / 2}(z)} \xi^{\mp k} e^{ \pm \xi / 2}[1], \\
& u_{h, j}^{\prime}(z)= \pm \lambda^{3 / 4} \phi^{1 / 2}(z) \xi^{\mp k} e^{ \pm \xi / 2}[1] .
\end{aligned}
$$

When $|\xi|>N$ but $z$ is in the domain $\Xi^{(m)}$, then the respective forms are

$$
\begin{aligned}
& u_{h, j}(z)=\frac{1}{\lambda^{1 / 4} \phi^{1 / 2}(z)}\left\{\left[A_{i, 1}^{(h, m)}\right] \xi^{-k} e^{\xi / 2}+\left[A_{i, 2}^{(h, m)}\right] \xi^{k} e^{-\xi / 2}\right\}, \\
& u_{h, j}^{\prime}(z)=\lambda^{3 / 4} \phi^{1 / 2}(z)\left\{\left[A_{j, 1}^{(h, m)}\right] \xi^{-k} e^{\xi / 2}-\left[A_{i, 2}^{(h, m)}\right] \xi^{k} e^{-\xi / 2}\right\},
\end{aligned}
$$

with coefficients given by the formulas (14) and (11).

It is sometimes possible to justify replacing a coefficient [0] by 0 itself on the ground that the forms given for two sectors $\Xi^{(h)}$ which have a region in common must be asymptotically equivalent within that region. Thus, by way of illustration, if every sector $\Xi^{(h)}$ consists of but two regions $r$, and there accordingly exist solutions having the forms (39) over the entire sectors $\Xi^{(h)}$, it is found that formulas (40) yield for $\xi$ in the sector $\Xi^{(-1)}$ the value

$$
u_{0,2}(z)=\frac{1}{\lambda^{1 / 4} \phi^{1 / 2}(z)}\left\{[0] \xi^{-k} e^{\xi / 2}+[1] \xi^{k} e^{-\xi / 2}\right\}
$$


Now unless the coefficient indicated as [0] is actually 0 , the term in which it occurs is dominant in the region common to $\Xi^{(-1)}$ and $\Xi^{(0)}$ and the formula conflicts with the appropriate formula (39). Hence in this case it must be concluded that the coefficient in question actually vanishes.

Lastly, when $|\xi| \leqq N$ these solutions may be described by the formulas

$$
\begin{aligned}
u_{h, j}(z)= & \frac{\mp 1}{\lambda^{1 / 4} \phi^{1 / 2}(z)}\left\{\Gamma\left(-\frac{1}{2}\right) C_{2,3-j}^{(h)} M_{k, 1 / 4}(\xi)+\Gamma\left(\frac{1}{2}\right) C_{1,3-j}^{(h)} M_{k,-1 / 4}(\xi)\right\} \\
& +\frac{E(z, \lambda) \log \lambda}{\lambda}, \\
u_{h, j}^{\prime}(z)= & \mp \lambda^{3 / 4} \phi^{1 / 2}(z)\left\{\Gamma\left(-\frac{1}{2}\right) C_{2,3-j}^{(h)}\left(M_{k, 1 / 4}^{\prime}(\xi)-\frac{\phi^{\prime}(z)}{2 \lambda \phi^{2}(z)} M_{k, 1 / 4}(\xi)\right)\right. \\
+ & \left.\Gamma\left(\frac{1}{2}\right) C_{1,3-j}^{(h)}\left(M_{k,-1 / 4}^{\prime}(\xi)-\frac{\phi^{\prime}(z)}{2 \lambda \phi^{2}(z)} M_{k,-1 / 4}(\xi)\right)\right\}+\frac{E(z, \lambda) \log \lambda}{\lambda} .
\end{aligned}
$$

UNIVERSITY OF WISCONSIN,

MAdisON, Wis. 